\title{
Erratum to: Mesenchymal stem cells attenuate hydrogen peroxide-induced oxidative stress and enhance neuroprotective effects in retinal ganglion cells
}

Yi Cui ${ }^{1} \cdot$ Nuo $\mathrm{Xu}^{2} \cdot$ Wei $\mathrm{Xu}^{3} \cdot$ Guoxing $\mathrm{Xu}^{3}$

Published online: 15 February 2017

(C) The Society for In Vitro Biology 2017

Erratum to: In Vitro Cell. Dev. Biol. Animal

DOI: 10.1007/s11626-016-0115-0

In the Acknowledgments section, the correct grant number of the Chinese Nature Science Foundation is 81271026.

The online version of the original article can be found at http://dx.doi.org/ 10.1007/s11626-016-0115-0.

$\triangle$ Guoxing Xu

fjmuxuguoxing@126.com

1 Department of Ophthalmology, Fujian Medical University Union Hospital, No.29, Xinquan Road, Gulou District, Fuzhou, Fujian Province 350001, China

2 Department of Ophthalmology, Fujian Provincial Hospital, No.134, East Street, Gulou District, Fuzhou, Fujian Province 350001, China

3 Department of Ophthalmology, Fujian Institute of Ophthalmology, The first Affiliated Hospital of Fujian Medical University, No.20, Chazhong Road, Taijiang District, Fuzhou, Fujian Province 350005, China 